\title{
Approximate Solutions, Thermodynamic Properties and Expectation Values With the Hua Plus Modified Eckart (HPME) Potential
}

\section{J. Njoku ( $\square$ ifeanyinjoku72@gmail.com )}

Federal University of Technology Owerri

C. J. Okereke

Federal University of Technology Owerri

C. P. Onyenegecha

Federal University of Technology Owerri

E. Onyeocha

Federal University of Technology Owerri

P. Nwaokafor

Federal University of Technology Owerri

F. U. Nwaneho

Federal University of Technology Owerri

\section{Research Article}

Keywords: Schrodinger equation, Formula method, Hua plus modified Eckart potential, Expectation values, Thermodynamic properties

Posted Date: September 29th, 2021

DOl: https://doi.org/10.21203/rs.3.rs-932748/v1

License: (c) (i) This work is licensed under a Creative Commons Attribution 4.0 International License.

Read Full License 
Approximate solutions, thermodynamic properties and expectation values with the Hua Plus modified Eckart (HPME) potential.

I. J. Njoku*, C.J. Okereke, C.P. Onyenegecha, E. Onyeocha, P. Nwaokafor, F. U. Nwaneho

Faculty of Sciences, Federal University of Technology Owerri, Nigeria.

"Corresponding Author Email address: ifeanyinjoku72@gmail.com (I.J. Njoku)

\begin{abstract}
The approximate solutions of Schrodinger equation for the Hua plus modified Eckart (HPME) potential is obtained via the Formula method. The vibrational partition function and other thermodynamic properties were investigated. Using the Hellmann-Feynman theorem, the expectation values of $r^{-2}, T$ and $p^{2}$ and their numerical values are also presented. Some cases of this potential are also studied. The results of our study are consistent with those in literature.

Keywords: Schrodinger equation; Formula method; Hua plus modified Eckart potential; Expectation values; Thermodynamic properties
\end{abstract}




\section{Introduction}

For many decades, the Schrodinger equation under various interactions, has been a subject of study owing to its applications in physical systems. A major task in such studies is to obtain exact solutions. As there are many systems which do not have exact solutions, approximate solutions, despite their drawbacks, are very essential. The approximate solutions of Schrodinger equation with different potentials have been reported [1-6]. One of such potentials is the Hua plus modified Eckart (HPME) potential [2, 3]. This potential is given as

$V(r)=V_{0}+V_{1}\left(\frac{1-e^{-2 \alpha r}}{1-q e^{-2 \alpha r}}\right)^{2}+V_{2}\left(\frac{4 e^{-2 \alpha r}}{\left(1-q e^{-2 \alpha r}\right)^{2}}\right)+V_{3}\left(\frac{1+e^{-2 \alpha r}}{1-q e^{-2 \alpha r}}\right)$,

where $V_{0}, V_{1}, V_{2}, V_{3}, \alpha, q$ are parameters. The HPME potential reduces to Eckart potential when $V_{0}$ $=V_{1}=0$ and $q=1$, Hua potential for $V_{0}=V_{2}=V_{3}=0$, and Poschl-Teller potential if $V_{0}=V_{1}=V_{3}$ $=0$ and $q=-1$. It is seen that the HPME potential incorporates Eckart potential, Hua potential and Poschl-Teller potential as special cases. Interesting reports on the special cases are also known [710], and clearly highlights their applications in various areas of physics [11-13]. Consequently, one can describe the HPME potential as an important realistic model in physics. Some authors have investigated this potential using different methods under Dirac, Schrodinger and KleinGordon equations [2,3, 14-15].

Recently, many researchers have studied thermodynamic properties [16-26] and expectation values [27-30] for different systems.

The objectives of this study are as follows: First, to solve the radial Schrodinger equation for the HPME potential via the formula approach [31]. Secondly, we study the thermodynamic properties of the HPME potential. Then, finally we obtain the expectation values of the HPME potential using the Hellmann-Feynman theorem [32,33]. This has not been reported in previous studies.

We prepare the work as follows: Section 2 contains a brief review of Formular method. The energy levels and eigenfunction are calculated in sec. 3. Sec. 4 gives the partition function and thermodynamic properties. In sect. 5, we evaluate the expectation values. Discussion of results appears in sec. 6. Finally, conclusion is presented in sec. 7.

\section{Formula Method}

Given the following second order hypergeometric ordinary differential equation of the form [34]

$$
\frac{d^{2} \psi(s)}{d s^{2}}+\frac{c_{1}-c_{2} s}{s\left(1-c_{3} s\right)} \frac{d \psi(s)}{d s}+\frac{\xi_{1} s^{2}+\xi_{2} s+\xi_{3}}{s^{2}\left(1-c_{2} s\right)^{2}} \psi(s)=0,
$$

where $\xi_{1}, \xi_{2}, \xi_{3}$ are constants. A method of solving such equation was proposed by Falaye et al. [31]. To derive the energy expression, the following condition is given 


$$
\left[\frac{c_{4}^{2}-c_{5}^{2}-\left[\frac{1-2 n}{2}-\frac{1}{2 c_{3}^{2}}\left(c_{2}-\sqrt{\left(c_{3}-c_{2}\right)^{2}-4 \xi_{1}}\right)\right]^{2}}{2\left[\frac{1-2 n}{2}-\frac{1}{2 c_{3}^{2}}\left(c_{2}-\sqrt{\left(c_{3}-c_{2}\right)^{2}-4 \xi_{1}}\right)\right]}\right]^{2}-c_{5}^{2}=0, c_{3} \neq 0,
$$

where,

$$
\left.\begin{array}{l}
c_{4}=\frac{\left(1-c_{1}\right)+\sqrt{\left(1-c_{1}\right)^{2}-4 \xi_{3}}}{2} \\
\left.c_{5}=\frac{1}{2}+\frac{c_{1}}{2}-\frac{c_{2}}{2 c_{3}}+\sqrt{\left(\frac{1}{2}+\frac{c_{1}}{2}-\frac{c_{2}}{2 c_{3}}\right)^{2}-\left(\frac{\xi_{1}}{c_{3}^{2}}+\frac{\xi_{2}}{c_{3}}+\xi_{3}\right)}\right)
\end{array}\right\}
$$

To obtain the wave function in terms of the generalized hypergeometric fucntions, we use

$\psi(s)=N_{n} s^{c_{4}}\left(1-c_{3} s\right)^{c_{5}} F_{1}\left(-n, n+2\left(c_{4}+c_{5}\right)+\frac{c_{2}}{c_{3}}-1 ; 2 c_{4}+c_{1} ; c_{3} s\right)$,

where $N_{n}$ is a normalization constant.

For the case where $c_{1}=c_{2}=c_{3}=1$, Onate et al. [35], put forward a simplified energy equation from eq. (3)

$$
\sqrt{-\xi_{3}}\left(1+\sqrt{1-4\left(\xi_{1}+\xi_{2}+\xi_{3}\right)}+2 n\right)=\xi_{3}-\xi_{1}-n(n+1)-n \sqrt{1-4\left(\xi_{1}+\xi_{2}+\xi_{3}\right)}-\left(\frac{1}{2}+\frac{1}{2} \sqrt{1-4\left(\xi_{1}+\xi_{2}+\xi_{3}\right)}\right)^{2} .
$$

\section{Schrodinger equation for HPME potential}

The Schrodinger equation in spherical coordinates is given in eq. (7) as [36]

$-\frac{\hbar^{2}}{2 \mu}\left[\frac{1}{r^{2}} \frac{\partial}{\partial r}\left(r^{2} \frac{\partial}{\partial r}\right)+\frac{1}{r^{2} \sin \theta} \frac{\partial}{\partial \theta}\left(\sin \theta \frac{\partial}{\partial \theta}\right)+\frac{1}{r^{2} \sin ^{2} \theta} \frac{\partial^{2}}{\partial \phi^{2}}\right] \Psi(r, \theta, \phi)=E_{n l} \Psi(r, \theta, \phi)$,

$E, \hbar, \mu$ and $\Psi$ are the energy, reduced Planck constant, reduced mass and wave function,

respectively. If we set $\Psi(r, \theta, \phi)=\frac{R(r)}{r} \psi_{n l}(\theta, \phi)$ into eq. (7), the equation reduces to a radial form given as

$\frac{d^{2} R_{n l}(r)}{d r^{2}}+\left[\frac{2 \mu}{\hbar^{2}}\left(E_{n l}-V(r)\right)-\frac{l(l+1)}{r^{2}}\right] R_{n l}(r)=0$,

where $n$ and $l$ are the radial and orbital angular momentum quantum numbers, respectively.

If we incorporate eq. (1) into (8), we obtain 
$\frac{d^{2} R_{n l}(r)}{d r^{2}}+\left[\frac{2 \mu}{\hbar^{2}}\left(E_{n l}-V_{0}-V_{1}\left(\frac{1-e^{-2 \alpha r}}{1-q e^{-2 \alpha r}}\right)-V_{2}\left(\frac{4 e^{-2 \alpha r}}{\left(1-q e^{-2 \alpha r}\right)^{2}}\right)-V_{3}\left(\frac{1+e^{-2 \alpha r}}{1-q e^{-2 \alpha r}}\right)\right)-\frac{l(l+1)}{r^{2}}\right] R_{n l}(r)=0 .(9)$

An exact solution to eq. (9) is not feasible due to $\frac{1}{r^{2}}$. Therefore, to solve (9), an approximation must be adopted for the centrifugal term [37]

$\frac{1}{r^{2}} \approx \frac{4 \alpha^{2} e^{-2 \alpha r}}{\left(1-q e^{-2 \alpha r}\right)^{2}}$

Using eq. (10) in (9) and making the transformation $s=q e^{-2 \alpha r}$, (9) becomes

$\frac{d^{2} R_{n l}(s)}{d s^{2}}+\frac{(1-s)}{s(1-s)} \frac{d R_{n l}(s)}{d s}+\frac{A s^{2}+B s+C}{s^{2}(1-s)^{2}} R_{n l}(s)=0$,

where

$$
\begin{aligned}
& A=\frac{\mu\left(E_{n l}-V_{0}-\frac{V_{1}}{q^{2}}\right)}{2 \alpha^{2} \hbar^{2}}, \\
& B=\frac{\mu\left(2 V_{0}-2 E_{n l}+\frac{2 V_{1}}{q}-\frac{4 V_{2}}{q}-\frac{V_{3}}{q}\right)}{2 \alpha^{2} \hbar^{2}}-\frac{l(l+1)}{q} \\
& C=\frac{\mu\left(E_{n l}-V_{0}-V_{1}-V_{3}\right)}{2 \alpha^{2} \hbar^{2}}
\end{aligned}
$$

Using the procedure outlined in section 2 above, the constants, $c_{1}=c_{2}=c_{3}=1$. Thus, we derive the constants in (4) as

$$
\left.\begin{array}{l}
c_{4}=\sqrt{-C} \\
c_{5}=\frac{1}{2}+\sqrt{\frac{1}{4}+\frac{l(l+1)}{q}+\frac{V_{1}(q-1)^{2}}{q}+\frac{4 V_{2}}{q}+\frac{V_{3}(q+1)}{q}}
\end{array}\right\} .
$$

Using the simplified energy expression in eq. (6), the energy expression of the HPME potential is given as 


$$
\begin{aligned}
& E_{n l}=V_{o}+V_{1}+V_{3} \\
& -\frac{2 \alpha^{2} \hbar^{2}}{\mu}\left(\frac{\frac{\mu\left(2 V_{1}(q-1)+4 V_{2}+V_{3}(1+2 q)\right)}{2 \alpha^{2} \hbar^{2}}+q n(n+1)+\frac{q}{2}+l(l+1)+\left(n+\frac{1}{2}\right) \sqrt{q^{2}+4 q l(l+1)+\frac{2 \mu\left(V_{1}(q-1)^{2}+4 q V_{2}+q V_{3}(1+q)\right)}{\alpha^{2} \hbar^{2}}}}{q+2 q n+\sqrt{q^{2}+4 q l(l+1)+\frac{2 \mu\left(V_{1}(q-1)^{2}+4 q V_{2}+q V_{3}(1+q)\right)}{\alpha^{2} \hbar^{2}}}}\right)
\end{aligned}
$$

The corresponding wave function is

$$
R_{n l}(s)=N_{n} s^{\sqrt{-C}}(1-s)^{c_{5}} F_{1}\left(-n, n+2\left(\sqrt{-C}+c_{5}\right) ; 2 \sqrt{-C}+1 ; s\right)
$$

\section{Thermodynamic properties of the HPME potential}

Setting $l=0$, the pure vibrational energy of the HPME potential is given as

$$
E_{n}=V-\frac{2 \alpha^{2} \hbar^{2}}{\mu}\left(\frac{Q}{2(n+\sigma)}+\frac{(n+\sigma)}{2}\right)^{2}
$$

where,

$$
\left.\begin{array}{l}
P=\frac{\mu}{2 \alpha^{2} \hbar^{2}}\left(2 V_{1}(q-1)+4 V_{2}+V_{3}(1+2 q)\right), \\
\sigma=\frac{1}{2}+\frac{1}{2 q} \sqrt{\frac{2 \mu}{\alpha^{2} \hbar^{2}}\left(V_{1}(q-1)^{2}+4 q V_{2}+q V_{3}(1+q)\right)+q^{2}} \\
Q=\frac{P+q \sigma(1-\sigma)}{q}, V=V_{0}+V_{1}+V_{3}
\end{array}\right\} .
$$

The partition function for the pure vibrational energy is gotten from the following expression

$$
Z(\beta)=\sum_{n=0}^{\lambda} e^{-\beta E_{n}}, \beta=\frac{1}{k_{B} T}
$$

where $k_{B}$ and $\beta$ are the Boltzmann constant and temperature parameter, respectively. $\mathrm{T}$ is the temperature and $\lambda$ is the upper bound quantum number, realised from $\left.\frac{d E_{n}}{d n}\right|_{n=\lambda}=0$.

$$
\lambda=\lfloor-\sigma \pm \sqrt{Q}\rfloor
$$

Using eq. (18), eq. (20) becomes

$$
Z(\beta)=\sum_{n=0}^{\lambda} \exp \left(-\beta\left(V-\frac{2 \alpha^{2} \hbar^{2}}{\mu}\left(\frac{(n+\sigma)}{2}+\frac{Q}{2(n+\sigma)}\right)^{2}\right)\right) .
$$

For classical thermodynamics, we set $\rho=n+\sigma$, the partition function becomes 
$Z(\beta)=\int_{\sigma}^{\lambda+\sigma} e^{Y \rho^{2} \beta-\frac{W}{\rho^{2}} \beta+X \beta} d \rho$

Evaluating eq. (23), the partition function becomes

$$
Z(\beta)=\frac{e^{\beta X} \sqrt{\pi}}{4 \Omega \sqrt{\beta Y}}\left(\Omega^{2}\left(\operatorname{erf}\left(\chi_{2}\right)-\operatorname{erf}\left(\chi_{1}\right)\right)+\operatorname{erf}\left(\chi_{2}\right)-\operatorname{erf}\left(\chi_{1}\right)\right),
$$

where

$$
\left.\begin{array}{l}
\Omega=e^{2 \sqrt{-\beta W} \sqrt{-\beta Y}} \\
\chi_{1}=\sigma \sqrt{-\beta Y}+\frac{\sqrt{-\beta W}}{\sigma} \\
\chi_{2}=(\sigma+\lambda) \sqrt{-\beta Y}+\frac{\sqrt{-\beta W}}{(\sigma+\lambda)} \\
X=\frac{\alpha^{2} \hbar^{2}}{\mu} Q-V \\
Y=\frac{\alpha^{2} \hbar^{2}}{2 \mu} \\
W=\frac{\alpha^{2} \hbar^{2}}{2 \mu} Q^{2} \\
\operatorname{erfi}(x)=\frac{\operatorname{erf}(i x)}{i}=\frac{2}{\sqrt{\pi}} \int_{0}^{x} e^{t^{2}} d t
\end{array}\right\}
$$

Other thermodynamic properties of the HPME are obtained as:

\section{Vibrational Internal Energy}

$U(\beta)=-\frac{\partial \ln Z(\beta)}{\partial \beta}$

\section{Vibrational Free Energy}

$$
F(\beta)=-\frac{1}{\beta} \ln Z(\beta)
$$

\section{Vibrational Entropy}

$$
S(\beta)=k \ln Z(\beta)-k \beta \frac{\partial \ln Z(\beta)}{\partial \beta} .
$$




\section{Vibrational Specific Heat Capacity}

$C(\beta)=k \beta^{2} \frac{\partial^{2} \ln Z(\beta)}{\partial \beta^{2}}$

\section{Expectation values of the HPME potential}

The expectation values of the HPME potential are obtained using the Hellmann-Feynman theorem $[32,33]$. The theorem states that the derivative of the energy of a system with respect to a parameter, $v$, is equal to the expectation value for the Hamiltonian with respect to the same parameter. The basic equation governing the Hellmann-Feynman theorem is given by

$$
\frac{\partial E_{n l}(v)}{\partial v}=\left\langle\psi_{n l}\left|\frac{\partial H(v)}{\partial v}\right| \psi_{n l}\right\rangle \text {. }
$$

The effective Hamiltonian for the HPME potential is

$$
H=\frac{-\hbar^{2}}{2 \mu} \frac{d^{2}}{d r^{2}}+\frac{\hbar^{2} l(l+1)}{2 \mu r^{2}}+V_{0}+V_{1}\left(\frac{1-e^{-2 \alpha r}}{1-q e^{-2 \alpha r}}\right)^{2}+V_{2}\left(\frac{4 e^{-2 \alpha r}}{\left(1-q e^{-2 \alpha r}\right)^{2}}\right)+V_{3}\left(\frac{1+e^{-2 \alpha r}}{1-q e^{-2 \alpha r}}\right)
$$

By setting $q$ to be $l$ and $\mu$, the expectation values for $r^{-2}$ and $T$, respectively are obtained. The expectation value $p^{2}$ is obtained using the formula $\langle T\rangle=\frac{\left\langle p^{2}\right\rangle}{2 \mu}$.

$$
\begin{aligned}
& \left\langle r^{-2}\right\rangle=\frac{8(q n)^{2}}{\hbar^{2} \delta \Delta(q+2 q n+\Delta)^{3}}\left[\begin{array}{l}
\left\{\delta\left(1+\frac{\Delta}{q n}\left(1+\frac{1}{2 n}\right)+\frac{l}{q n^{2}}(1+l)+\frac{1}{2 n^{2}}\right)+2 \mu+\frac{3 \phi}{4}\right\} \\
\times\left\{\delta\left(\frac{\Delta}{q^{2} n}\left(1+\frac{1}{2 n}\right)+\frac{l}{(q n)^{2}}(1+l)+\frac{1}{q}\left(1+\frac{1}{n}+\frac{1}{2 n^{2}}\right)\right)+\phi+3 \mu\right\}
\end{array}\right]
\end{aligned}
$$

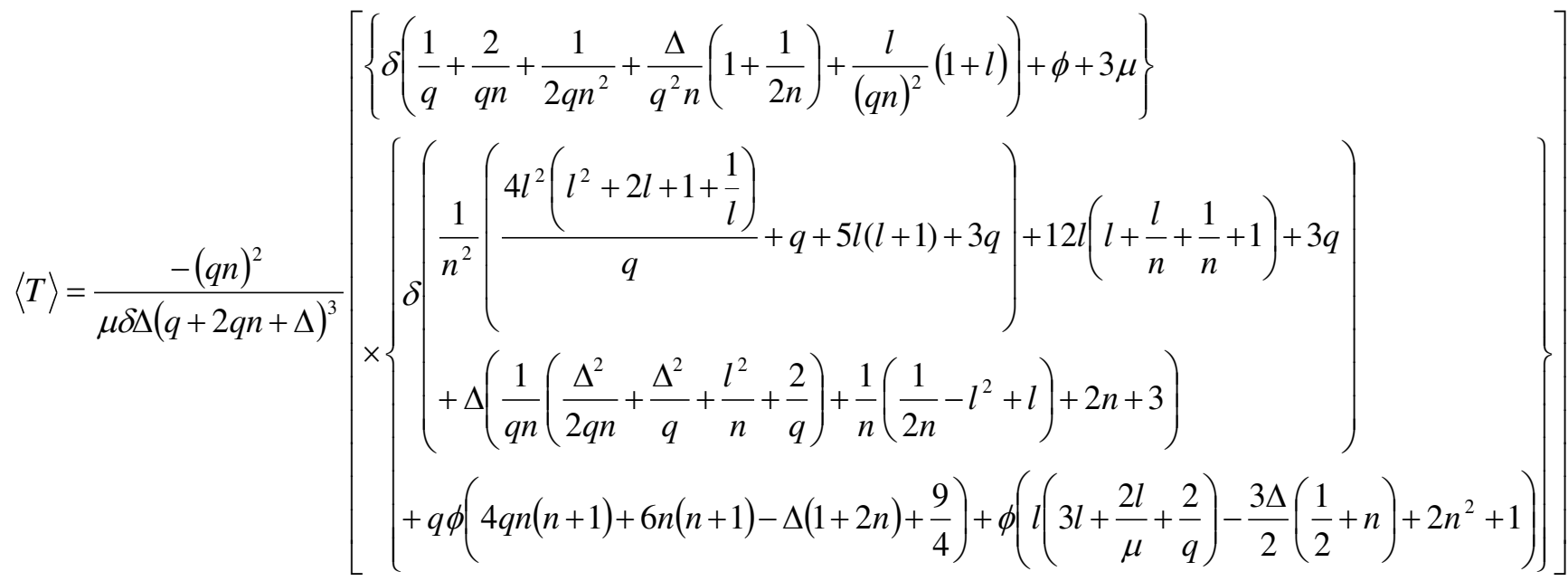




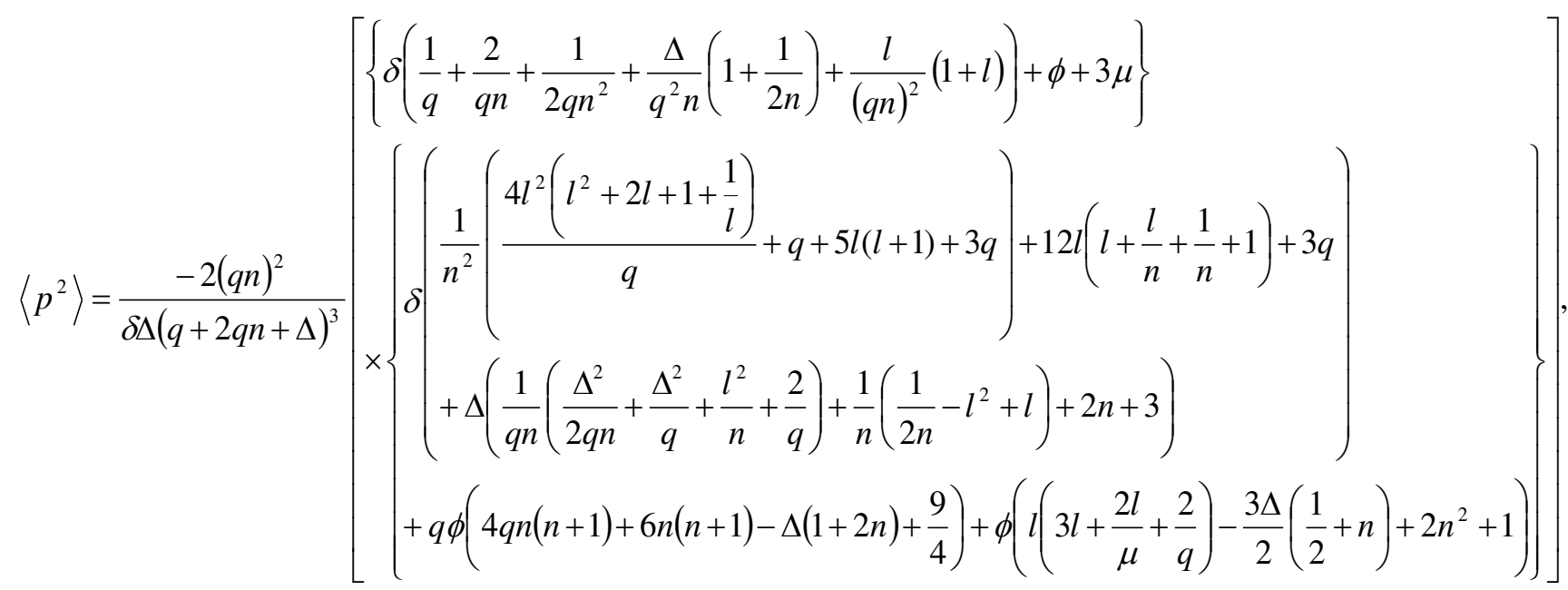

where,

$$
\left.\begin{array}{l}
\delta=2(\alpha \hbar n q)^{2} \\
\Delta=\sqrt{q^{2}+4 q l(l+1)+\frac{2 \mu\left((q-1)^{2}+4 q+q(1+q)\right)}{\alpha^{2} \hbar^{2}}} \\
\phi=4 \mu q
\end{array}\right\} .
$$

\section{Discussion of Results}

Table 1 gives numerical results of the energy of the HPME potential. The energy is observed to increase as quantum numbers $n$ and $l$ increase for $\alpha=0.02$. For $\alpha=0.04$ and $\alpha=0.06$, a similar trend is observed up to $n=4$, for $\alpha=0.04$ and $n=3$ for $\alpha=0.06$, when the energy is observed to decrease as $n$ and $l$ increase. In Tables 2 and 3, the energies of the Hua and modified Eckart potentials are observed to respectively increase as $n$ and $l$ increase. Table 4 gives the numerical results of the energy of the HPME potential for the exact case $(l=0)$. The results are seen to be in agreement with results presented in the literature [3]. The slight variation can be attributed to the approximation used in this study for the centrifugal term. This is a validation of the accuracy of our result. In table 5, the expectation values of $r^{-2}, T$ and $p^{2} .\left\langle r^{-2}\right\rangle$ is observed to increase with increasing values of $n$ and $l$. On the other hand $\langle T\rangle$ and $\left\langle p^{2}\right\rangle$ are observed to decrease as $n$ and $l$ increase. 


\begin{tabular}{lllll}
0 & 0 & -5.266622496 & -5.248221248 & -5.230507222 \\
1 & 0 & -5.231021126 & -5.183233712 & -5.141885066 \\
2 & 0 & -5.198529476 & -5.129781682 & -5.077401056 \\
& 1 & -5.197749730 & -5.127375336 & -5.073422772 \\
3 & 0 & -5.168993044 & -5.086793584 & -5.033904078 \\
& 1 & -5.168285848 & -5.084888414 & -5.031398536 \\
& 2 & -5.166877596 & -5.081158460 & -5.026720226 \\
4 & 0 & -5.142268178 & -5.053338510 & -5.008824958 \\
& 1 & -5.141630044 & -5.051890962 & -5.007618236 \\
& 2 & -5.140359588 & -5.049068068 & -5.005489420 \\
& 3 & -5.138468368 & -5.045011236 & -5.002982046 \\
5 & 0 & -5.118221184 & -5.028604624 & -5.000052112 \\
& 1 & -5.117648868 & -5.027576624 & -5.000000648 \\
& 2 & -5.116509728 & -5.025585608 & -5.000141662 \\
& 3 & -5.114814720 & -5.022758826 & -5.000941248 \\
& 4 & -5.112580096 & -5.019280634 & -5.003047726 \\
\hline
\end{tabular}

Table 4

Energy eigenvalues of the HPME potential with $V_{0}=2.5 ; V_{1}=0.3 ; V_{2}=-0.05 ; V_{3}=0.4 ; q=0.09$; $\mu=0.5 ; \hbar=1 ; l=0$

\begin{tabular}{lcc}
\hline $\boldsymbol{n}$ & Present study & Hassanabadi et al. [3] \\
\hline 0 & 2.932998240 & 3.1024 \\
1 & 2.954999940 & 3.1152 \\
2 & 2.975979327 & 3.1270 \\
3 & 2.995947646 & 3.13792 \\
4 & 3.014915950 & 3.1478 \\
5 & 3.032895106 & 3.15695 \\
6 & 3.049895796 & 3.1651 \\
7 & 3.065928522 & 3.1724 \\
8 & 3.081003609 & 3.1788 \\
9 & 3.095131210 & 3.1843 \\
\hline
\end{tabular}




\section{Table 5}

Numerical results of the expectation values of the HPME potential with $V_{0}=0.2$,

\begin{tabular}{ccccc}
$V_{1}=0.3, V_{2}=0.4 ; V_{3}=0.5, q=0.6, \alpha=0.01, \mu=\hbar=1$ & \\
\hline $\boldsymbol{n}$ & $\boldsymbol{l}$ & $\left\langle r^{-2}\right\rangle\left(\begin{array}{l}o^{-2} \\
A\end{array}\right)$ & $\langle T\rangle(\mathrm{eV})$ & $\left\langle p^{2}\right\rangle(e V / c)^{2}$ \\
& & & & \\
\hline 0 & 0 & 0.0001659961078 & -0.005517931840 & -0.01103586368 \\
1 & 0 & 0.0001667639368 & -0.01660535193 & -0.03321070386 \\
2 & 0 & 0.0001675315157 & -0.02779459478 & -0.05558918957 \\
& 1 & 0.0001675315893 & -0.02796308568 & -0.05592617137 \\
3 & 0 & 0.0001682988499 & -0.03908561192 & -0.07817122384 \\
& 1 & 0.0001682988954 & -0.03925525346 & -0.07851050692 \\
& 2 & 0.0001682989866 & -0.03959453656 & -0.07918907312 \\
4 & 0 & 0.0001690659447 & -0.05047835625 & -0.1009567125 \\
& 1 & 0.0001690659622 & -0.05064914790 & -0.1012982957 \\
& 2 & 0.0001690659977 & -0.05099073100 & -0.1019814620 \\
& 3 & 0.0001690660505 & -0.05150310565 & -0.1030062113 \\
5 & 0 & 0.0001698328054 & -0.06197278205 & -0.1239455640 \\
& 1 & 0.0001698327951 & -0.06214472325 & -0.1242894466 \\
& 2 & 0.0001698327748 & -0.06248860560 & -0.1249772111 \\
& 3 & 0.0001698327440 & -0.06300442850 & -0.1260088569 \\
& 4 & 0.0001698327030 & -0.06369219075 & -0.1273843815 \\
\hline
\end{tabular}

In figs. 1(a-f), the energy of the HPME potential is plotted against various potential parameters for various values of $n$ and $l=1$. In figs. $1 \mathrm{a} \& \mathrm{~b}$, the energy is observed to decrease as the screening parameter, $\alpha$ and deformation parameter, $q$, increase, respectively. In figs. $1 \mathrm{c} \& \mathrm{~d}$, the energy is seen to increase in a monotonic manner as $V_{0}$ and $V_{l}$ increase, respectively. In fig. $1 \mathrm{e}$, the energy decreases as $V_{2}$ increases, while, in fig. 1f, energy increases as $V_{3}$ increases.

In fig. $2 \mathrm{a}$, the vibrational partition function, $\mathrm{Z}$, is observed to increase as the parameter, $\beta$, increases, i.e., as temperature decreases. The implication of this is that as the temperature drops, the probability that the system occupies a given microstate also decreases. In fig. $2 \mathrm{~b}, \mathrm{Z}$ is seen to first increase slowly, then sharply as the upper bound vibrational quantum number, $\lambda$, increases. In figs. $3 \mathrm{a} \& \mathrm{~b}$, respectively, the vibrational internal energy, $\mathrm{U}$, increases sharply as $\beta$ and $\lambda$ increase, but cuts off at a point for all values of $\lambda$ and $\beta$. In fig. $4 \mathrm{a}$, the vibrational free energy, $\mathrm{F}$, of the system is observed to increase as $\beta$ increases up to about $\beta=0.01$, then begins to approach to a constant value. In fig. $4 \mathrm{~b}, \mathrm{~F}$ is observed to decrease as $\lambda$ increases. In figs. $5 \mathrm{a} \& \mathrm{~b}$, the vibrational entropy, $\mathrm{S}$, of the system first increases slowly, then sharply rises as both $\beta$ and $\lambda$ respectively increase. For the vibrational specific heat capacity, $C$, as can be seen in figs. $6 a \& b$, there is an increase in $\mathrm{C}$ with respective increase in $\beta$ and $\lambda$. 


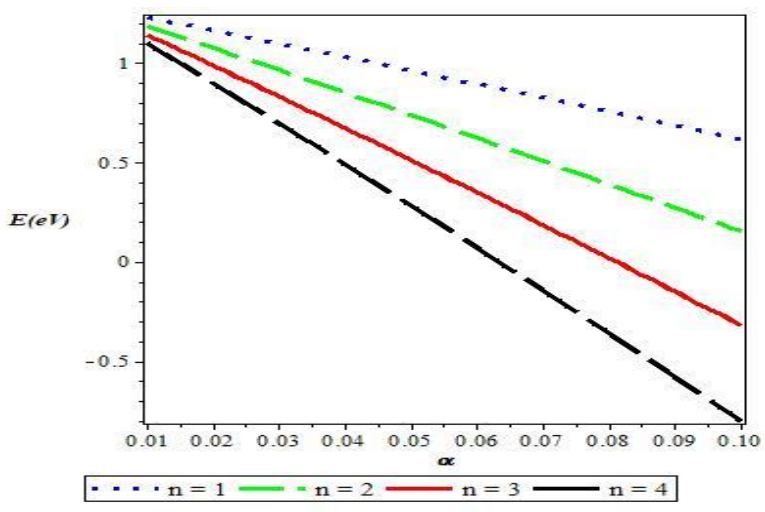

(a)

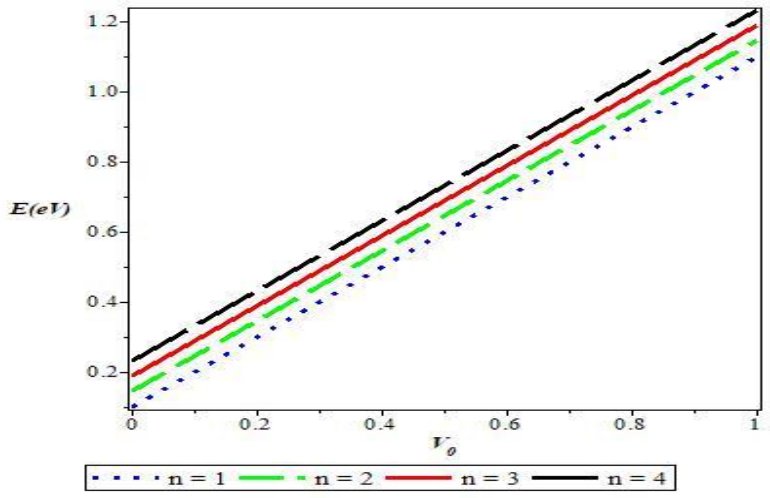

(c)

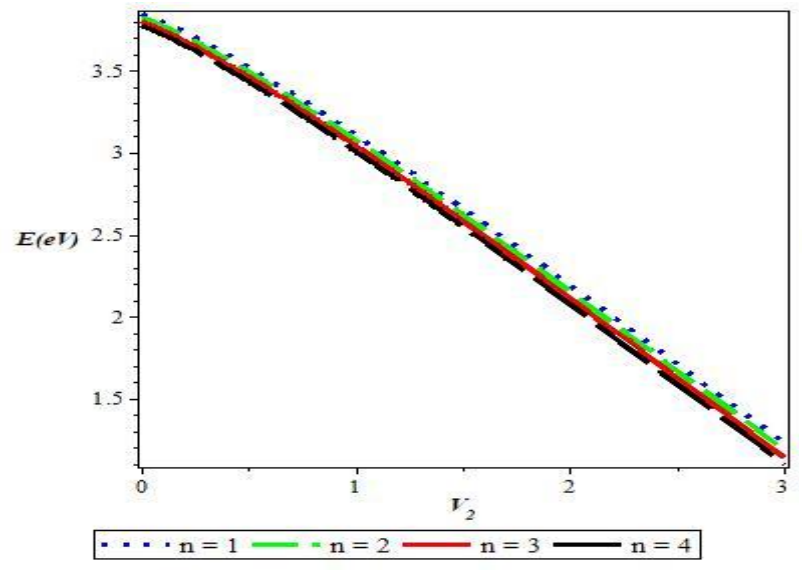

(e)

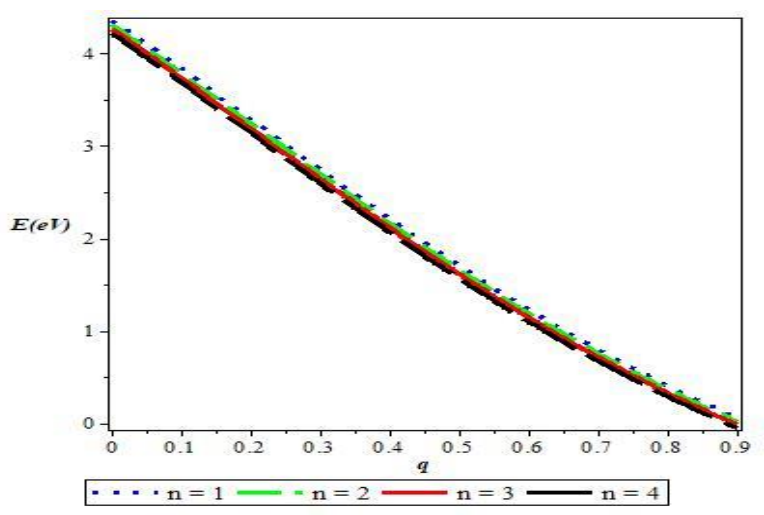

(b)

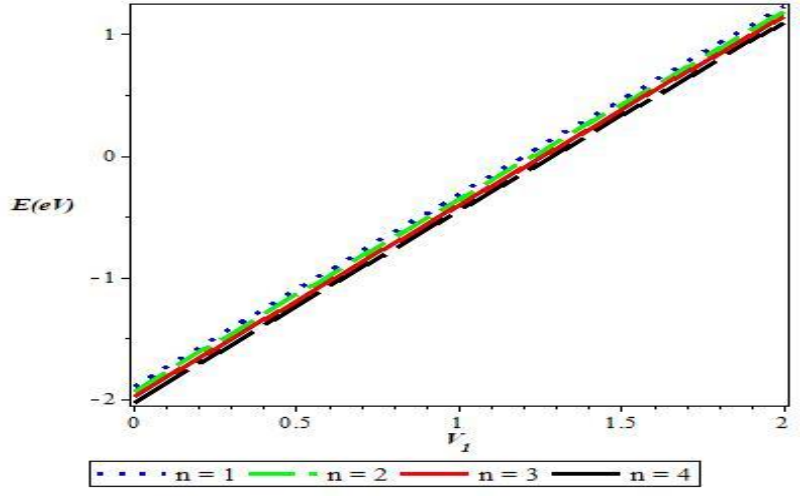

(d)

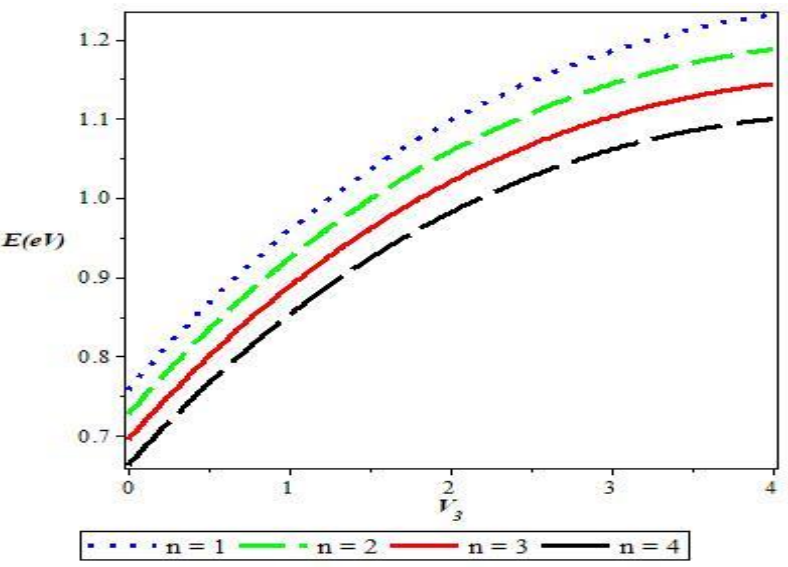

(f)

Fig. 1. Variation of Energy of the HPME potential with various potential parameters with $V_{0}=1, V_{l}=2$, $V_{2}=3, V_{3}=4, l=1, q=0.6$ and $\alpha=0.01$ 


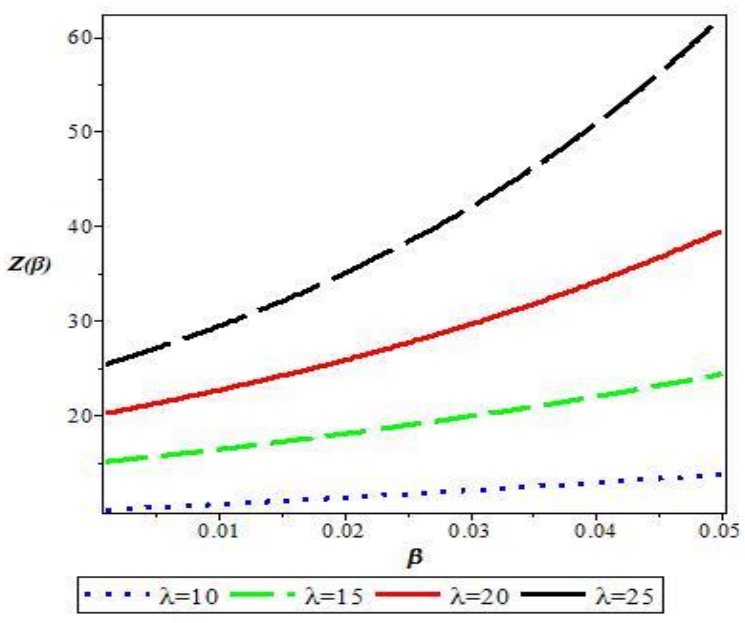

(a)

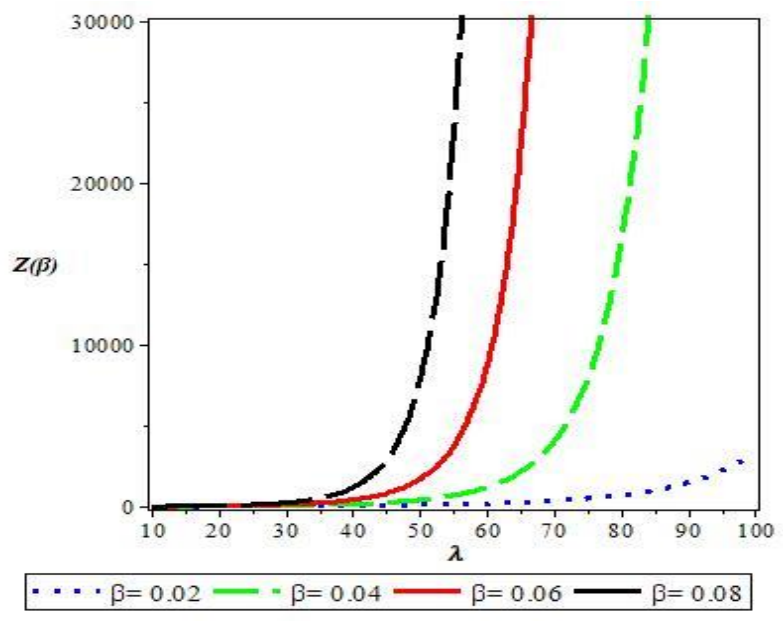

(b)

Fig. 2. Variation of vibrational partition function of the HPME potential with $\beta$ and $\lambda$ with $V_{0}=1, V_{l}=2$, $V_{2}=3, V_{3}=4, l=1, q=0.6$ and $\alpha=0.2$

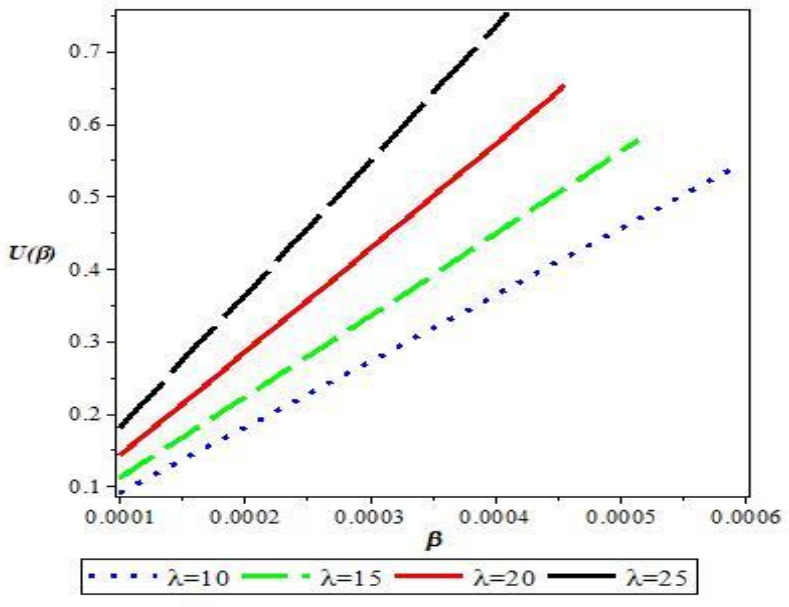

(a)

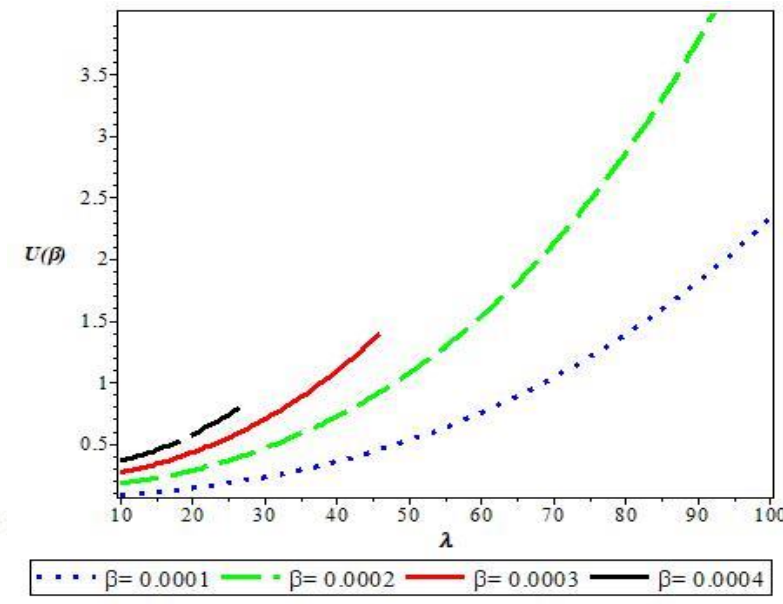

(b)

Fig. 3. Variation of vibrational internal energy of the HPME potential with $\beta$ and $\lambda$ with $V_{0}=1, V_{l}=2$,

$$
V_{2}=3, V_{3}=4, l=1, q=0.6 \text { and } \alpha=0.2
$$




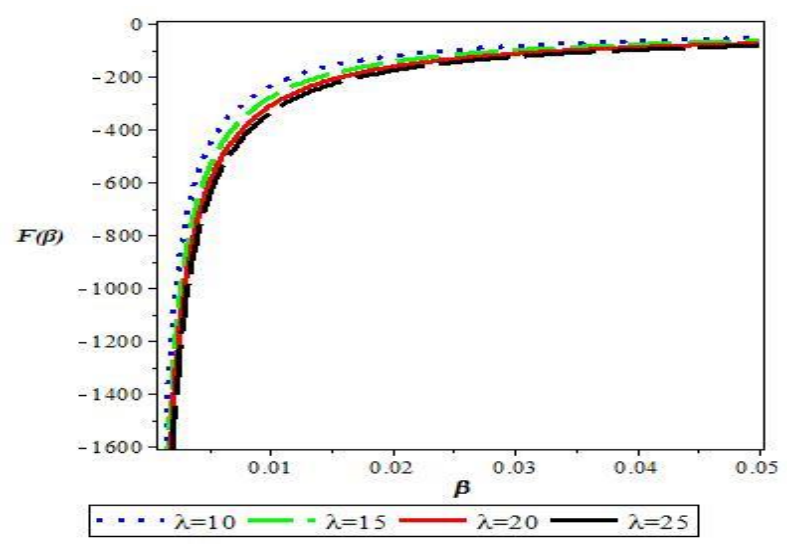

(a)

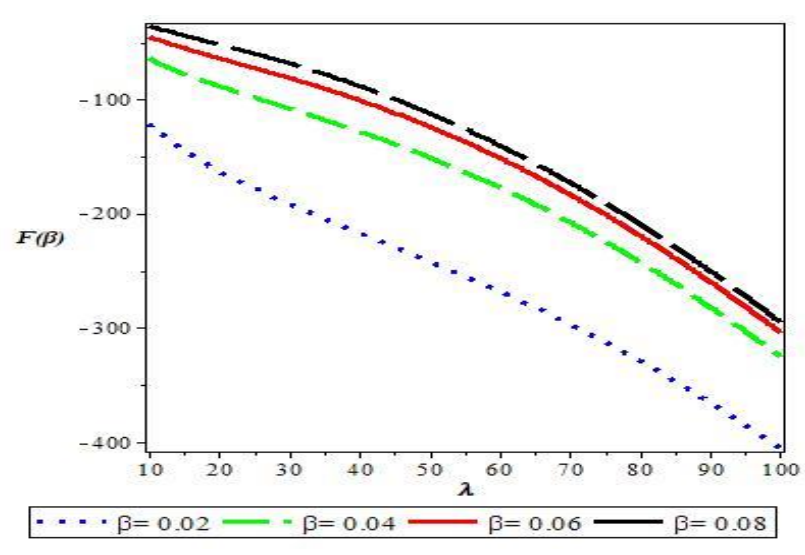

(b)

Fig. 4. Variation of vibrational free energy of the HPME potential with $\beta$ and $\lambda$ with $V_{0}=1, V_{l}=2, V_{2}=3$, $V_{3}=4, l=1, q=0.6$ and $\alpha=0.2$

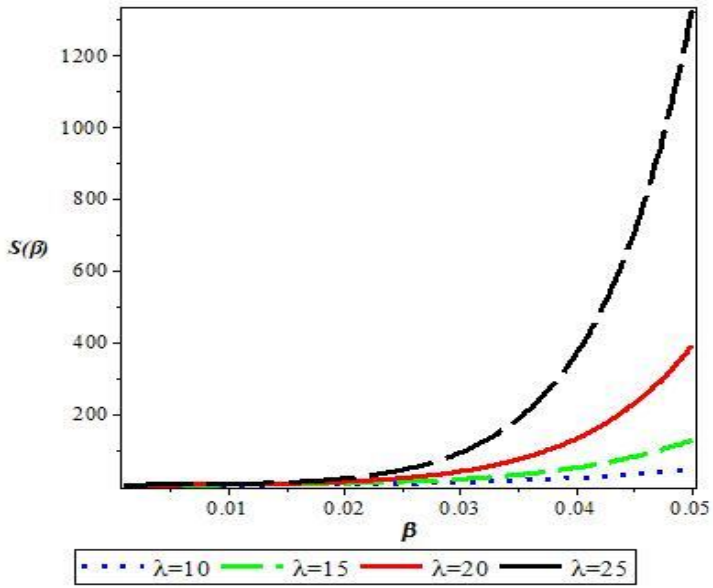

(a)

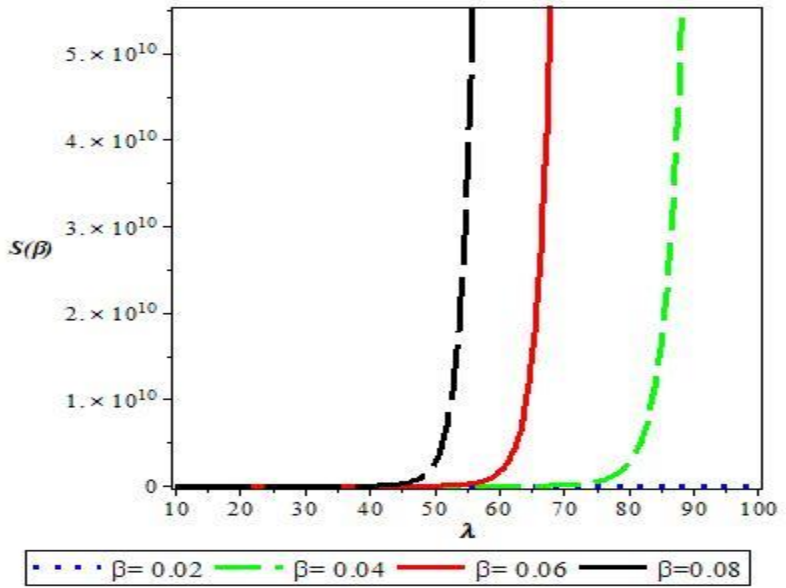

(b)

Fig. 5. Variation of vibrational entropy of the HPME potential with $\beta$ and $\lambda$ with $V_{0}=1, V_{l}=2, V_{2}=3, V_{3}=4$, $l=1, q=0.6$ and $\alpha=0.2$ 


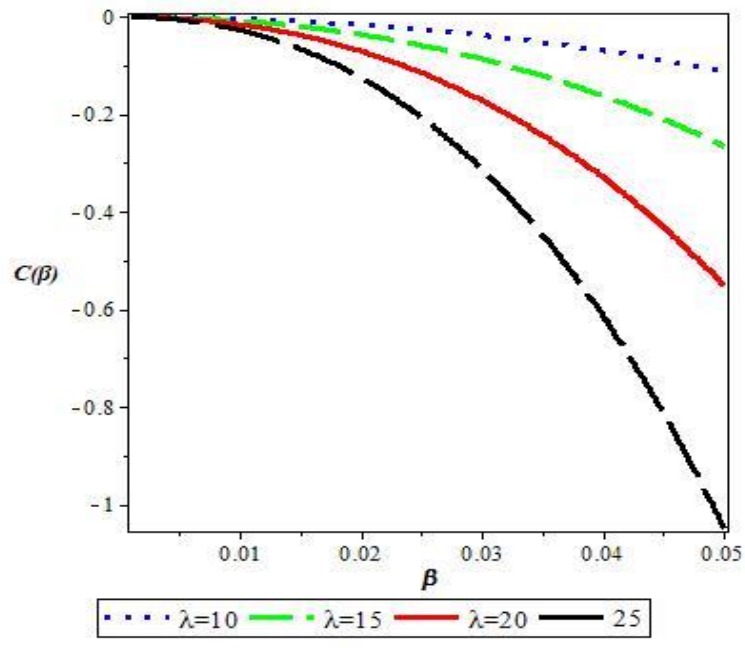

(a)

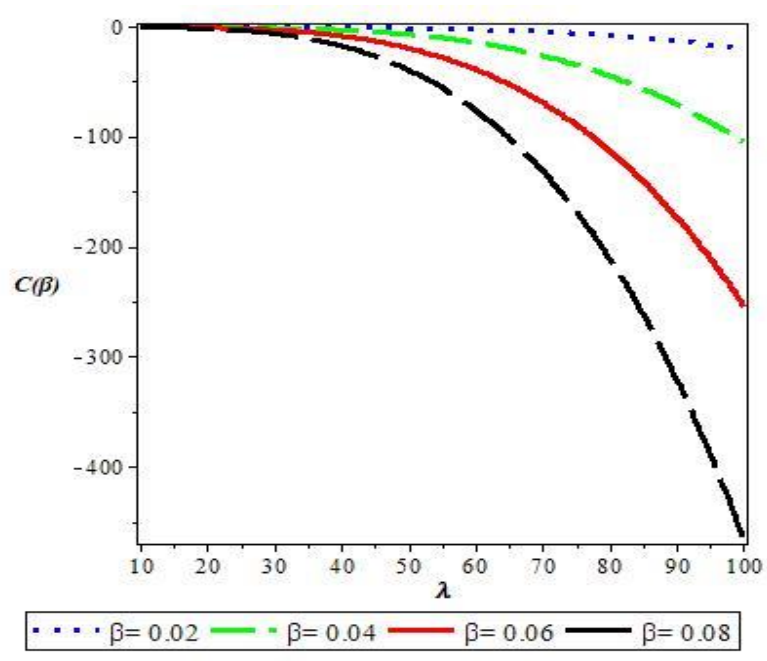

(b)

Fig. 6. Variation of vibrational specific heat capacity of the HPME potential with $\beta$ and $\lambda$ with $V_{0}=1$, $V_{l}=2, V_{2}=3, V_{3}=4, l=1, q=0.6$ and $\alpha=0.2$

\subsection{Special cases}

\section{Case 1: Modified Eckart Potential}

If we make $V_{0}=V_{l}=0, q=1$, the HPME potential reduces to the modified Eckart potential

$V(r)=V_{2}\left(\frac{4 e^{-2 \alpha r}}{1-q e^{-2 \alpha r}}\right)+V_{3}\left(\frac{1+e^{-2 \alpha r}}{1-q e^{-2 \alpha r}}\right)$.

Thus, (16) reduces to

$$
E=V_{3}-\frac{2 \alpha^{2} \hbar^{2}}{\mu}\left(\frac{\frac{\mu}{2 \alpha^{2} \hbar^{2}}\left(4 V_{2}+3 V_{3}\right)+\frac{1}{2}+n(n+1)+l(l+1)+\left(n+\frac{1}{2}\right) \sqrt{\frac{8 \mu\left(V_{2}+V_{3}\right)}{\alpha^{2} \hbar^{2}}+(2 l+1)^{2}}}{1+2 n+\sqrt{\frac{8 \mu\left(V_{2}+V_{3}\right)}{\alpha^{2} \hbar^{2}}+(2 l+1)^{2}}}\right)^{2} .
$$

\section{Case 2: Hua Potential}

If we set $V_{0}=V_{2}=V_{3}=0$, the HPME potential reduces to the Hua potential

$V(r)=V_{1}\left(\frac{1-e^{-2 \alpha r}}{1-q e^{-2 \alpha r}}\right)^{2}$

Consequently, (16) becomes 
$E=V_{1}-\frac{2 \alpha^{2} \hbar^{2}}{\mu}\left(\frac{\frac{\mu V_{1}(q-1)}{2 \alpha^{2} \hbar^{2}}+\frac{q}{2}+q n(n+1)+l(l+1)+\left(n+\frac{1}{2}\right) \sqrt{\frac{2 \mu V_{1}(q-1)^{2}}{\alpha^{2} \hbar^{2}}+4 q l(l+1)+q^{2}}}{q+2 q n+\sqrt{\frac{2 \mu V_{1}(q-1)^{2}}{\alpha^{2} \hbar^{2}}+4 q l(l+1)+q^{2}}}\right)^{2}$.

If we set $\alpha r=b_{h}\left(r-r_{e}\right)$ and using the approximation $\frac{1}{r^{2}} \approx \frac{1}{r_{e}}\left(D_{0}+\frac{D_{1} e^{-b_{h}\left(r-r_{e}\right)}}{1-q e^{-b_{h}\left(r-r_{e}\right)}}+\frac{D_{2} e^{-2 b_{h}\left(r-r_{e}\right)}}{\left(1-q e^{-b_{h}\left(r-r_{e}\right)}\right)^{2}}\right)$

, the energy in eq. (39) will become exactly the same as eq. (14) in ref. [17].

Case 4: Poschl-Teller Potential

Adjusting $V_{0}=V_{l}=V_{3}=0, q=-1$, the HPME potential reduces to the Poschl-Teller potential

$V(r)=V_{2}\left(\frac{4 e^{-2 \alpha r}}{1+e^{-2 \alpha r}}\right)$.

Hence, (16) reduces to

$$
E=-\frac{2 \alpha^{2} \hbar^{2}}{\mu}\left(\frac{\frac{-\mu V_{2}}{\alpha^{2} \hbar^{2}}+\frac{1}{2}+n(n+1)-l(l+1)-\left(n+\frac{1}{2}\right) \sqrt{1-4 l(l+1)+\frac{8 \mu V_{2}}{\alpha^{2} \hbar^{2}}}}{1+2 n-\sqrt{1-4 l(l+1)+\frac{8 \mu V_{2}}{\alpha^{2} \hbar^{2}}}}\right)^{2} .
$$

If we set $V_{2}=-V_{l}$, eq. (41) will be similar to eq. (20) in Ref. [38]. Also, if we set $q=1$ and $l=0$, the energy (16) becomes very identical to eq. (32) in ref. [3].

\section{Conclusions}

In this paper, we solved the Schrodinger equation for the HPME potential via the Formula method. In addition, we obtain the partition function and other thermodynamic properties of the HPME potential. Furthermore, the expectation values of the HPME potential were determined using the powerful Hellmann-Feynman theorem. Numerical values of the energies and the expectation values are presented. The results of the study are in good agreement with literature.

\section{References}

[1] C.P. Onyenegecha, C.A. Onate,O.K. Echendu, A.A. Ibe, H. Hassanabadi, Solutions of Schrodinger equation for the modified Mobius square plus Kratzer potential, Eur. Phys. J. Plus 135(2020)289.

[2] C.P. Onyenegecha, U.M. Ukewuihe, A.I. Opara, C.B. Agbakwuru, C.J. Okereke, N.R. Ugochukwu, S.A. Okolie, I.J. Njoku, Approximate solutions of Schrödinger equation for the Hua plus modified Eckart potential with the centrifugal term. Eur. Phys. J. Plus 135 (2020) 571. 
[3] H. Hassanabadi, B.H. Yazarloo, A.N. Ikot, N. Salehi, S. Zarrinkamar, Exact analytical versus numerical solutions of Schrodinger equation for Hua plus modified Eckart potential, Ind. J Phys. 87 (2013) 1219.

[4] R. Rani, S.B. Bhardwaj, F. Chand, Bound state solutions to the Schrödinger equation for some diatomic molecules, Pram. J. Phys. 91 (2018) 46.

[5] O. Bayrak, I. Boztosun, Bound state solutions of the Hulthén potential by using the asymptotic iteration method, Phys. Scr. 76 (2007) 92.

[6] O. Bayrak, M. Karakoc, I. Boztosun, R. Sever, Analytical Solution of the Schrödinger equation for Makarov potential with any L Angular Momentum, Int. J. Theor. Phys. 47 (2008) 3005.

[7] F. Taskin, G. Kocak, Approximate solutions of Schrodinger equation for Eckart potential with centrifugal term, Chin. Phys. B 19 (2010) 090314.

[8] M. Hamzavi, A.A. Rajabi H. Hassanabadi, The rotation-vibration spectrum of diatomic molecules with the Tietz-Hua rotating oscillator and approximation scheme to the centrifugal term, Mol. Phys. 110, (2012) 389.

[9] Y. Xu, S.He, C. S. Jia, Approximate analytical solutions of the Dirac equation with the PöschlTeller potential includingthe spin-orbit coupling term, J. Phys. A: Math. Gen.41 (2008) 255302.

[10] G.F. Wei, S.H. Dong, A novel algebraic approach to spin symmetry for Dirac equation with scalar and vector second Pöschl-Teller potentials, Eur. Phys. J. A43 (2010) 185.

[11] M. Eshghi, S. M. Ikhdair, Dirac particle in generalized Pöschl-Teller field including a Coulomb-like tensor coupling: super-symmetric solution, Math. Meth. Appl. Sci. 37 (2014) 2829.

[12] F. cooper, A. Kahare, U. Sukhatme, Supersymmetry and quantum mechanics, Phys. Rept. 251 (1995) 267.

[13] W. Hua, Four-parameter exactly solvable potential for diatomic molecules Phys. Rev. A 24 (1990) 2524.

[14] H. Hassanabadi, B.H. Yazarloo, S. Zarrinkamar, Exact Solution of Klein-Gordon Equation for Hua Plus Modified Eckart Potentials, Few-Body Syst. 54 (2013) 2017.

[15] A.N. Ikot, E. Maghsoodi, A.D. Antia, H. Hassanabadi, S. Zarrinkamar. Approximate Solutions of the Dirac Equation for the Hua Plus Modified Eckart Potential. Arab. J Sci. Eng. 40 (2015) 2063.

[16] U.S. Okorie, A.N. Ikot, E.O. Chukwuocha, M.C. Onyeaju, P.O. Amadi, M.J. Sithole, G.J. Rampho. Energies Spectra and Thermodynamic Properties of Hyperbolic Pöschl-Teller Potential (HPTP) model, Int. J. Thermophys. 41 (2020) 91.

[17] I.J. Njoku, C.P. Onyenegecha, C.J. Okereke, A.I. Opara, U.M. Ukewuihe, F.U. Nwaneho, Approximate solutions of Schrodinger equation and thermodynamic properties with Hua potential. Res. Phys. 24 (2021) 104208. 
[18] H. Louis, B.I. Ita, N.I. Nzeata, Approximate solution of the Schrodinger equation with Manning-Rosen plus Hellmann potential and its thermodynamic properties using the proper quantization rule, Eur. Phys. J. Plus 134 (2019) 315.

[19] U.S. Okorie, A.N. Ikot, E.O. Chukwuocha, G.J. Rampho, Thermodynamic properties of improved deformed exponential-type potential (IDEP) for some diatomic molecules, Res. Phys. 17 (2020) 103078.

[20] U.S. Okorie, A.N Ikot, M.C. Onyeaju, E. O. Chukwuocha. Bound state solutions of Schrödinger equation with modified Mobius square potential (MMSP) and its thermodynamic properties. J. Mol. Mod. 24 (2018) 289.

[21]_H. Hassanabadi, S. Sargolzaeipor, B. H. Yazarloo, Thermodynamic Properties of the ThreeDimensional Dirac Oscillator with Aharonov-Bohm Field and Magnetic Monopole Potential, Few-Body Syst. 56 (2015) 115.

[22] E.S. Eyube, J.B. Yerima, A.D. Ahmed, J-state solutions and thermodynamic properties of the Tietz oscillator. Phys. Scr. 96 (2021) 055001

[23]_.B. Okon, O.O. Popoola, E. Omugbe, A.D. Antia, C.N. Isonguyo, E.E. Ituen. Thermodynamic properties and bound state solutions of Schrodinger equation with Mobius square plus screenedKratzer potential using Nikiforov-Uvarov method, Comp. Theor. Chem. 1196 (2021) 113132.

[24] K.J. Oyewumi, W.A. Yahya, Thermodynamic properties and approximate solutions of the $l$ state Pöschl-Teller-type potential J. Assoc. Arab Univers. Basic Appl. Scien., 21 (2015) 53.

[25] K.J. Oyewumi, W.A. Yahya, Thermodynamic properties and approximate solutions of the $l$ state Pöschl-Teller-type potential J. Assoc. Arab Univers. Basic Appl. Sci. 21 (2015)53.

[26] S. H. Dong, M. Cruz-Irisson, Energy spectrum for a modified Rosen-Morse potential solved by proper quantization rule and its thermodynamic properties, J. Math. Chem. 50 (2012) 881.

[27] I. B. Okon, O. Popoola, C. N. Isonguyo, Approximate Solutions of Schrodinger Equation with Some Diatomic Molecular Interactions Using Nikiforov-Uvarov Method, Adv. High Energy Phys. $2(2017) 1$.

[28] I.B. Okon, A. D. Antia, L.E. Akpabio, B.U. Archibong, Expectation values of some diatomic molecules with Deng-fan potential using hellmann-feynman theorem, J. Appl. Sci. Intl. 10 (2018) 247.

[29] A.N. Ikot, U.S. Okorie, R. Sever, G.J. Rampho, Eigensolution, expectation values and thermodynamic properties of the screened Kratzer potential, Eur. Phys. J. Plus 134 (2019) 386

[30] O.J. Oluwadare, K.J. Oyewumi, Energy spectra and the expectation values of diatomic molecules confined by the shifted Deng-Fan potential, Eur. Phys. J. Plus 133 (2018) 422. 
[31] B.J. Falaye, S.M. Ikhdair, M. Hamzavi, Formula method for bound state problems, Few-Body Syst. 56 (2014) 63.

[32] R.P. Feynman, Forces in Molecules, Phys. Rev. 56 (1939) 340.

[33] H. Hellmann, On the role of the kinetic electron energy in the interatomic forces, Zeit. Phys. 85 (1933) 180.

[34] C. Tezcan, R. Sever, A general approach for the exact solution of the Schrödinger equation, Int. J. Theor. Phys. 48 (2009) 337.

[35] Onate, C.A., Adebimpe, O., Lukman, A.F., Adama, I.J., Davids, E.O., Dopamu, K.O.: Approximate solutions of the Dirac equation with Coulomb-Hulthén-like tensor interaction. Res. Phys. 11, 1094 (2018). https://doi.org/10.1016/j.rinp.2018.10.052

[36] L.D. Landau, E.M. Lifshitz, Quantum Mechanics-Non-Relativistic Theory, (Pergamon: Oxford), 1977.

[37] R.L. Greene, C. Aldrich, Variational wave functions for a screened Coulomb potential. Phys, Rev. A. 143 (1976) 2363.

[38]_A.D. Antia, I.B. Okon, A.O. Akankpo, J. B. Usanga, Non-Relativistic Bound state solutions of Modified Quadratic Yukawa plus $q$-Deformed Eckart Potential, J. App. Math. Phys. 8 (2020) 660.

\section{Author contribution statement}

IJN: conceptualization of the manuscript and wrote the manuscript. CJO and CPO: performed the calculations and wrote the manuscript. EO and PN: prepared all the figures and took part in the investigation. FUN: prepared all the tables and edited the manuscript. All the authors reviewed the manuscript.

\section{Additional Information}

\section{Competing Interest}

The authors declare no conflict of interest. 